


\title{
Particle Tracking Code for Simulating Global RF Feedback
}

\author{
L. K. Mestha \\ Superconducting Super Collider Laboratory ${ }^{\dagger}$ \\ 2550 Beckleymeade Ave. \\ Dallas, TX 75237
}

September 1991

TOperated by the Universities Research Association, Inc., for the U.S. Department of Energy under Contract No. DE-AC35-89ER40486.

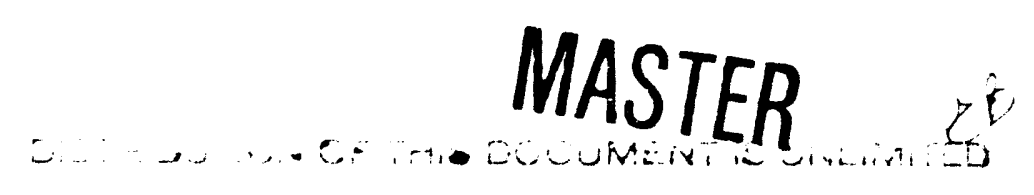




\section{CONTENTS}

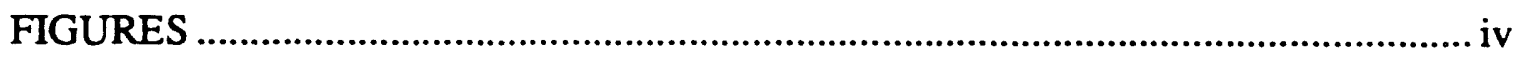

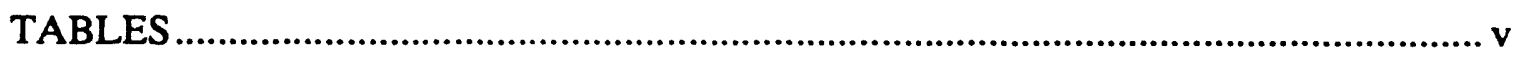

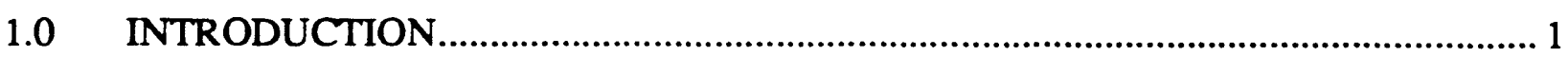

2.0 ONE-PARTICLE TRACKING MODEL _....................................................... 1

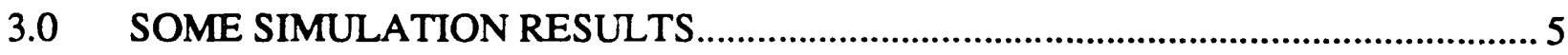

4.0 RF BEAM FEEDBACK LOOPS .............................................................. 13

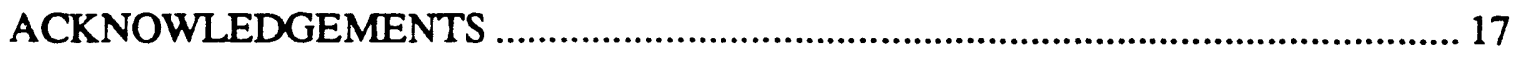

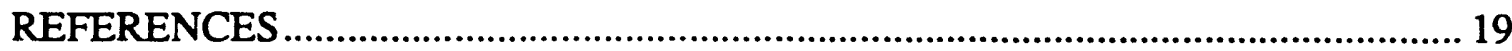




\section{FIGURES}

1. Schematic Representation of the Actual Orbit and the Equivalent Circular Orbit ........... 1

2. Schematic Representation of the Circular Orbit with One Accelerating Gap.................. 1

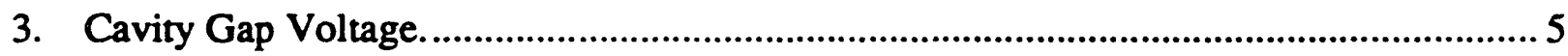

4. Particle Phase Predicted Using the Tracking Code of Table 1....................................... 5

5. Fortran Code for Tracking One Particle........................................................................ 6

6. Synchronous Phase As Calculated from ESME....................................................... 7

7. Particle Phase Predicted Using Thin Element Tracking Code......................................... 7

8. Phase Curves of Figures 4 and 7 Shown with Expanded Scale...................................... 7

9. Time Variation of the $B$-Field Error Signal..............................................................8

10. Particle Phase Predicted Using the Codes of Figure 5 with B-Error. ............................. 8

11. Particle Phase Predicted Using Thin Element Tracking Code with B-Error. .................. 9

12. Phase Curves of Figures 10 and 11 Shown with Expanded Scale. .................................9

13. Mean Radial Orbit Shift Predicted Using Codes Shown in Figure 5

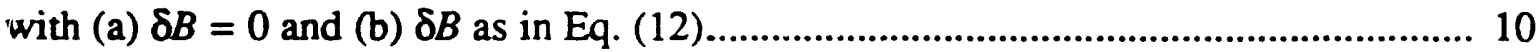

14. Mean Radial Orbit Shift Predicted Using Thin Element Tracking Code with (a) $\delta B=0$ and (b) $\delta B$ as in Eq. (12)................................................. 10

15. Variation of Mean Radial Orbit with Respect to Particle Phase Using Codes Shown in Figure 5 (No Field Error Included). ........................................ 10

16. Variation of Mean Radial Orbit with Respect to Particle Phase Using Thin Element Tracking Code (No Field Error Included). ................................. 10

17. Variation of Mean Radial Orbit with Respect to Phase Using Thin Element Tracking Code When the Particle Is Launched with $3 \sigma$ in Both Transverse Planes.

18. Phase Curves When the Particle Is Launched with $3 \sigma$ in Both Transverse Planes (Expanded Scale of Figures 4 and 17).

19. Phase Curves When the Particle Is Launched at +10 deg.

20. Particle Phase with Respect to Time When the $B$-Field Is Held at Minimum $B$ for $50 \mathrm{~ms}$ (a) Using the One-Particle Tracking Code and

(b) Using the Thin Element Tracking Code. 13

21. Phase Curve When 1 Out of 12 Cavities Breaks Down at 30 ms. ................................. 13

22. Phase Curve with a Voltage Error of $50 \mathrm{KHz}$ Bandwidth and $1 \%$ Amplitude Deviation

23. Conceptual Diagram of the Various if Beam Feedback Loops with One if Cavity. 


\section{TABLES}

1. One Particle Tracking Model ........................................................................... 4 


\subsection{INTRODUCTION}

It is well know in the "control community" that a good feedback controller design is deeply rooted in the physics of the system. For example, when accelerating the beam we must keep several parameters under control so that the beam travels within the confined space. Important parameters include the frequency and phase of the if signal, the dipole field, and the cavity voltage. Because errors in these parameters will progressively mislead the beam from its projected path in the tube, feedback loops are used to correct the behavior. Since the feedback loop feeds energy to the system, it changes the overall behavior of the sy'stem and may drive it to instability. Various types of controllers are used to stabilize the feedback loop. Integrating the beam physics with the feedback controllers allows us to carefully analyze the beam behavior. This will not only guarantee optimal performance but will also significantly enhance the ability of the beam control engineer to deal effectively with the interaction of various feedback loops. Motivated by this theme, we developed a simple one-particle tracking code to simulate particle behavior with feedback controllers.

In order to achieve our fundamental objective, we can ask some key questions: What are the input and output parameters? How can they be applied to the practical machine? How can one interface the rf system dynamics such as the transfer characteristics of the if cavities and phasing between the cavities? Answers to these questions can be found by considering a simple case of a single cavity with one particle, tracking it tum-by-tum with appropriate initial conditions, then introducing constraints on crucial parameters. Critical parameters are if frequency, phase, and amplitude once the dipole field has been given. These are arranged in the tracking code so that we can interface the feedback system controlling them. Detailed simulation with the associated $\mathrm{rf}$ feedback loops affecting the beam is not shown in this report due mainly to the sheer volume of technology involved in this task. However, at the end of this repor, a conceptual diagram of the loops is shown with one if cavity. This would become the basis for modeling and simulating the beam behavior when integrated with the system.

\subsection{ONE-PARTICLE TRACKING MODEL}

Consider a circular accelerator whose real orbit, whatever its shape, is replaced by circles with circumferences equal to the actual path lengths, as shown in Figure 1. For mathematical considerations let us use a simple case of a single accelerating cavity with an infinitesimally small gap length insened in the beam line, as shown in Figure 2. The particle travelling in the beam line will see accelerating field only at the pointlike gap and will see no field elsewhere. Analogous to the accelerating case we also consider thin magnetic lenses such that the magnetic field will be maintained constant for one complete traversal across the gap. The magnetic field will be changed each time at the exit from the gap along the particle orbit. Later we will see that the representations devised are considerably accurate for modeling the Low Energy Booster (LEB) global rf feedback.

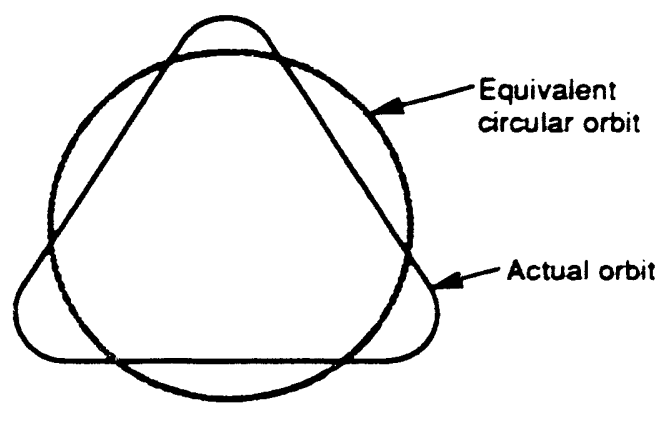

Figure 1. Schematic Representation of the Actual Orblt and the Equivalent Circular Orbit.

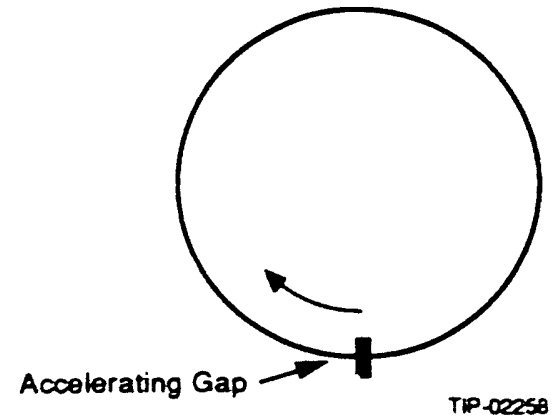

Figure 2. Schematic Representation of the Circular Orblt with One Accolerating Gap. 
Now, if the particle with initial energy of $E_{0}$ is injected at the gap location at a phase $\phi_{0}$ with respect to the zero crossover of the altemating gap voltage of peak amplitude $V_{k}$, then the acceleration takes place when it travels out of the gap. For a pointlike cavity, the phase $\phi_{0}$ is the same before entering the gap as it is just after leaving the gap, $\phi k$. Hence the particle would have acquired an additional energy of $\Delta E_{k}$ equal to

$$
\Delta E_{k}=V_{k} \sin \phi_{k}
$$

The suffix $k$ in Eq. (1) represents the parameter updates while the particles are travelling in the $k$ th tum. With this convention $E_{0}$ can be replaced by $E_{k-1}$. Thus the total energy after passing through the gap is

$$
E_{k}=E_{k-1}+\Delta E_{k}
$$

It is recalled at this stage that while determining the energy gain in the kth traversal chrough the gap, the peak value of the amplitude-modulated gap voltage is changed only at the gap location and remains unchanged elsewhere.

The actual momentum $P_{k}$ after acquiring energy $\Delta E_{k}$ is calculated from the following equation:

$$
P_{k}=\frac{1}{c} \sqrt{E_{k}^{2}-m^{2} c^{4}}
$$

where $m$ is the rest mass of the proton and $c$ is the speed of light.

So far, we have not introduced the synchronous particle into the model. The concept of the synchronous particle is very useful in describing the particle motion during acceleration, although there is no such particle in practice. As is well known about accelerators, the s!nchronous particle is always at the phase-stable position at every pass through the gap. The phase-stable position of the accelerating particle is chosen on the rising side of the sinusoidal gap voltage below transition energy. A particle which has too much energy travels in a circle with greater radius, but faster hence takes a shorter time to retum to the gap than the synchronous particle. Since the momentum of the synchronous particle is known, we first find the traversal time required for such a particle having a synchronous energy of $E_{k}$ in the kth pass and later work out the deviations in arrival time of the non-synchronous particle. To determine the time taken by the synchronous particle to complete the first tum, we must know its correct energy. This is given by

$$
E_{k}^{s}=\sqrt{\left(P_{k}^{s} c\right)^{2}+\left(m c^{2}\right)^{2}}
$$

where $P_{k}^{s}$ is calculated from the synchronous momentum curve at $\mathrm{t}=0$, just before crossing the gap for the first time. The traversal time $t_{k}$ of the synchronous particle for one complete tum is determined by the velocity in the $k$ th $t u m$. The velocity can be determined from the synchronous momentum and energy of the particle. Thus,

$$
\tau_{k}^{s}=\frac{C^{s}}{v_{k}^{s}} \text { where } v_{k}^{s}=\frac{P_{k}^{s} c^{2}}{E_{k}^{s}}
$$

The particle which has a momentum other than that of the synchronous particle takes a different time to arrive at the gap. 'The particle which is not at the synchronous phase of the accelerating wave will have a position error of, say, $\delta C_{k}$. The deviation is also due to errors in the bending magnetic field. If $\delta \tau_{k}$ s the 
deviation in time with respect to the time taken by the synchronous particle, then we can write the following equation: ${ }^{1}$

$$
\frac{\delta \tau_{k}}{\tau_{k}^{s}}=\left(\frac{1}{\gamma_{T}^{2}}-\frac{1}{\left(\gamma_{k}^{s}\right)^{2}}\right) \frac{\delta P_{k}}{P_{k}^{s}}-\frac{1}{\gamma_{T}^{2}} \frac{\delta B_{k}}{B_{k}^{s}},
$$

where $\delta P_{k}=P_{k}-\stackrel{S}{\not k}$ is the momentum difference and $\delta B_{k}=B_{k}-B_{k}^{5}$ is the field error as seen by the particle under consideration with reference to the ideal case. The factor $\psi_{k}^{5}$ is obtained for the synchronous particle from Eq. (5). We have used the momentum compaction factor $\left(1 / \gamma^{2} T\right)$, which represents the measure of the change in path length for a given fractional change in momentum. Also, in obtaining the above equations we have decoupled the betatron oscillations. This approximation, for the purpose of rf feedback, introduces very little error if the periods of betatron and synchrotron oscillations differ a great deal. This treatment has been confirmed by comparing the results of the tracking code with transverse dynamics (TEAPOT-like) for the LEB. ${ }^{2}$

From Eqs. (5) and (6) the total traversal time of any given particle is

$$
\tau_{k}=\tau_{k}^{s}+\delta \tau_{k}
$$

Since the gap is altemating with a frequency of $f_{k}$, then the phase covered by the particle in retuming to the cavity is given by

$$
\phi_{k+1}=\phi_{k}+2 \pi f_{k} \tau_{k}
$$

More accurate representation of the phase is obtained by integrating the ramping frequency in small, equal time steps (say, $m$ ) in one tum, as shown in Eq. (9):

$$
\phi_{k+1}=\phi_{k}+2 \pi \sum_{n=1}^{m} f(t) \frac{\tau_{k}}{m} .
$$

The new phase $\phi_{k+1}$ is used to compute the energy gain for the $k+1$ th tum and so on. The energy $E_{k-1}$ in Eq. (2) is replaced by $k$ to continue the calculations for the $k+1$ th turn. Furthermore, the radial orbit of the beam is important for the the global if feedback because the change in frequency, phase, or amplitude of the gap voltage would lead to orbital errors in addition to the errors in the bending magnetic field. The average radial orbit shift with respect to the synchronous orbit for each traversal can be obtained by using the well-known equation

$$
\frac{\delta R_{k}}{R^{s}}=\frac{1}{\gamma_{T}^{2}}\left(\frac{\delta P_{k}^{R}}{P_{k}^{s}}-\frac{\delta B_{k}}{B_{k}^{s}}\right)
$$


where $R^{S}$ represents the radius of the circle with circumference equal to the actual path length of the synchronous orbit, and the momentum difference is the same as that used to calculate the additional traversal time in Eq. (6). When the magnetic field error is zero, the momentum difference should altemate with a mean value equal to zero, meaning that the particle is always following the synchronous particle. In our calculation we change the momentum of the particle after leaving the cavity; just before leaving, the particle has a momentum equal to the momentum we calculated when the particle left the cavity in the previous tum. In contrast, the synchronous particle momentum is changing continuously. Hence an average of the momenta before entering and after leaving the cavity would be an accurate representation by which to calculate the mean radial orbit deviation as shown by Eq. (11). The large momentum change per turn in a machine like the LEB would add errors in the calculation of the mean orbit deviation.

$$
\delta P_{k}^{R}=\left(\frac{P_{k}+P_{k}-1}{2}\right)-P_{k}^{s} .
$$

Table 1 summarizes all the equations used in the tracking model. With this model, it is clear that we have access to $r i t$ parameters such as $V_{k}, f_{k}$, and $\phi_{k}$, and we can simulate the beam oscillations with errors in them. Also, magnetic field errors can be introduced if we know the error band in the field signal. These topics are covered later in the section on feedback loops. But first, we will discuss validation tests required to confirm the tracking model.

TABLE 1. ONE PARTICLE TRACKING MODEL.

$\begin{array}{lll}\text { Energy: } & \Delta E_{k}=V_{k} \sin \phi_{k} & \\ & E_{k}=E_{k-1}+\Delta E_{k} & \\ \text { Momentum: } & P_{k}=\frac{1}{c} \sqrt{E_{k}^{2}-m^{2} c^{4}} & \\ & \delta P_{k}=P_{k}-P_{k}^{s} & \left.\delta P_{k}^{R}=\left(\frac{P_{k}+P_{k}-1}{2}\right)-P_{k}^{s} c\right)^{2}+\left(m c^{2}\right)^{2} \\ & \end{array}$

Traversal Time:

$$
\begin{array}{ll}
\tau_{k}=\tau_{k}^{s}+\delta \tau_{k} & \\
\tau_{k}^{s}=\frac{C^{s}}{v_{k}^{s}} & v_{k}^{s}=\frac{P_{k}^{s} c^{2}}{E_{k}^{s}} \\
\delta \tau_{k}=\tau_{k}^{s}\left(\left(\frac{1}{\gamma_{T}^{2}}-\frac{1}{\left(\gamma_{k}^{s}\right)^{2}}\right) \frac{\delta P_{k}}{P_{k}^{s}}-\frac{1}{\gamma_{T}^{2}} \frac{\delta B_{k}}{B_{k}^{s}}\right)
\end{array}
$$

Beam Phase:

$$
\phi_{k+1}=\phi_{k}+2 \pi f_{k} \tau_{k}
$$

or

$$
\phi_{k+1}=\phi_{k}+2 \pi \sum_{n=1}^{m} f(t) \frac{\tau_{k}}{m}
$$

Mecin Radial Orbit:

$$
\delta R_{k}=R^{s}\left(\frac{1}{\gamma_{T}^{2}}\left(\frac{\delta P_{k}^{R}}{P_{k}^{s}}-\frac{\delta B_{k}}{B_{k}^{s}}\right)\right)
$$




\subsection{SOME SIMULATION RESULTS}

Using the model shown in Table 1 we tracked one particle for the LEB at $t=0$ to $0.05 \mathrm{sec}$ with the voltage function shown in Figure 3. Figure 4 shows the plot of the particle phase with respect to time for $E_{0}=600 \mathrm{MeV}$ and $\phi_{0}=0$, and with all other injection parameters in the program shown in Figure 5. Synchronous phase calculated from ESME particle-tracking code using the synchronous particle concept is shown in Figure 6. Figure 7 shows the plot of the phase with respect to time as obtained from the Thin Element Particle Tracking Codes by going through each magnetic lens at $5 \mathrm{~ns}$ time step. Figure 8 shows the expanded view of the comparison tetween Figures 7 and 4 . In our code, the simulations are done with the frequency updates equally divided by 114 steps within each turn, as compared to integration of the frequency ramping at intervals of $1.9 \mu \mathrm{s}$ in the Thin Element Tracking Codes. Clearly, the phase values coincide very well.

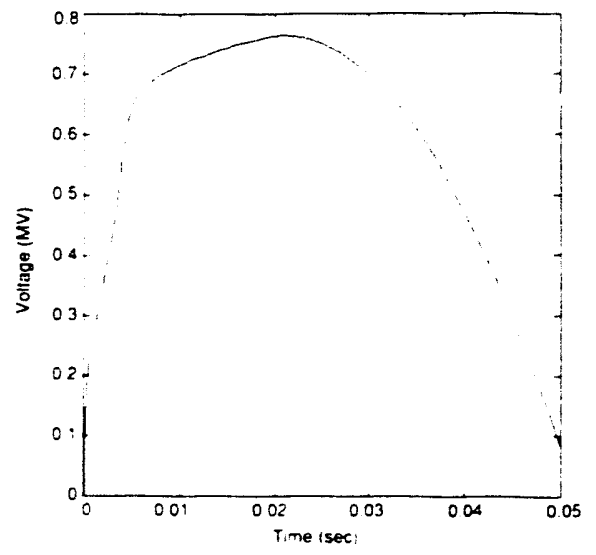

Figure 3. Cavity Gap Voltage.

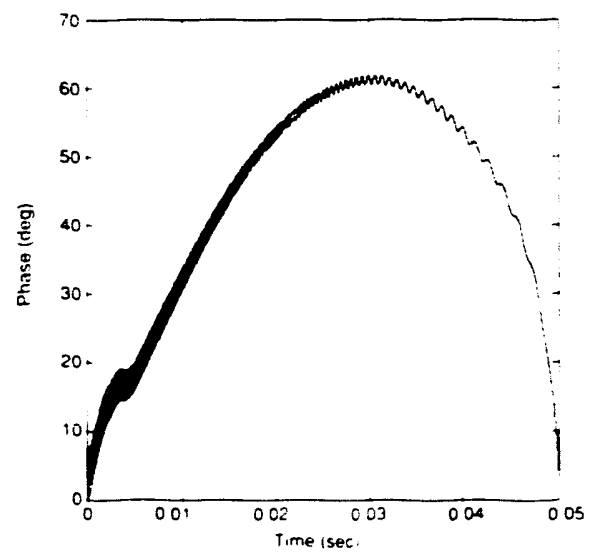

Figure 4. Particle Phase Predicted Using the Tracking Code of Table 1. 


\begin{tabular}{|c|c|c|c|}
\hline c & 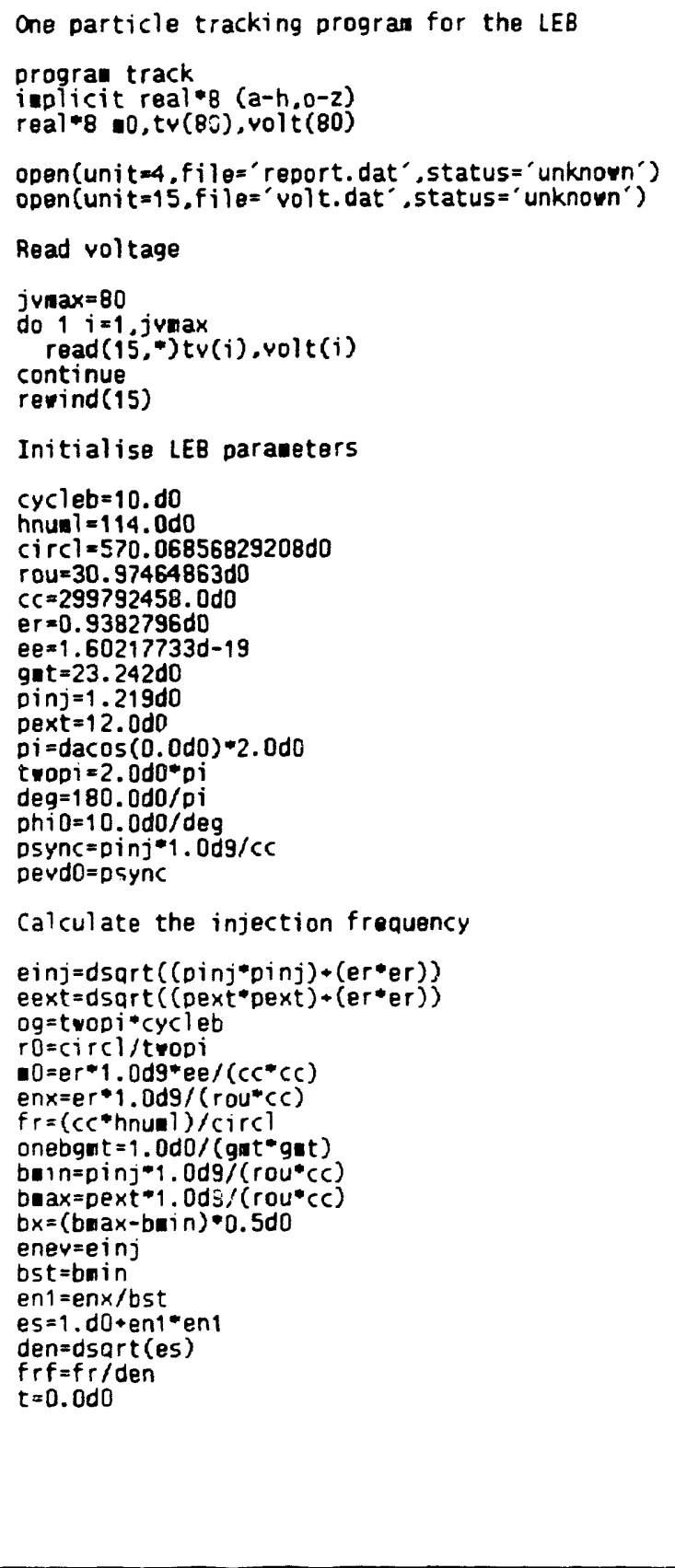 & $\begin{array}{l}c \\
51 \\
c \\
c\end{array}$ & 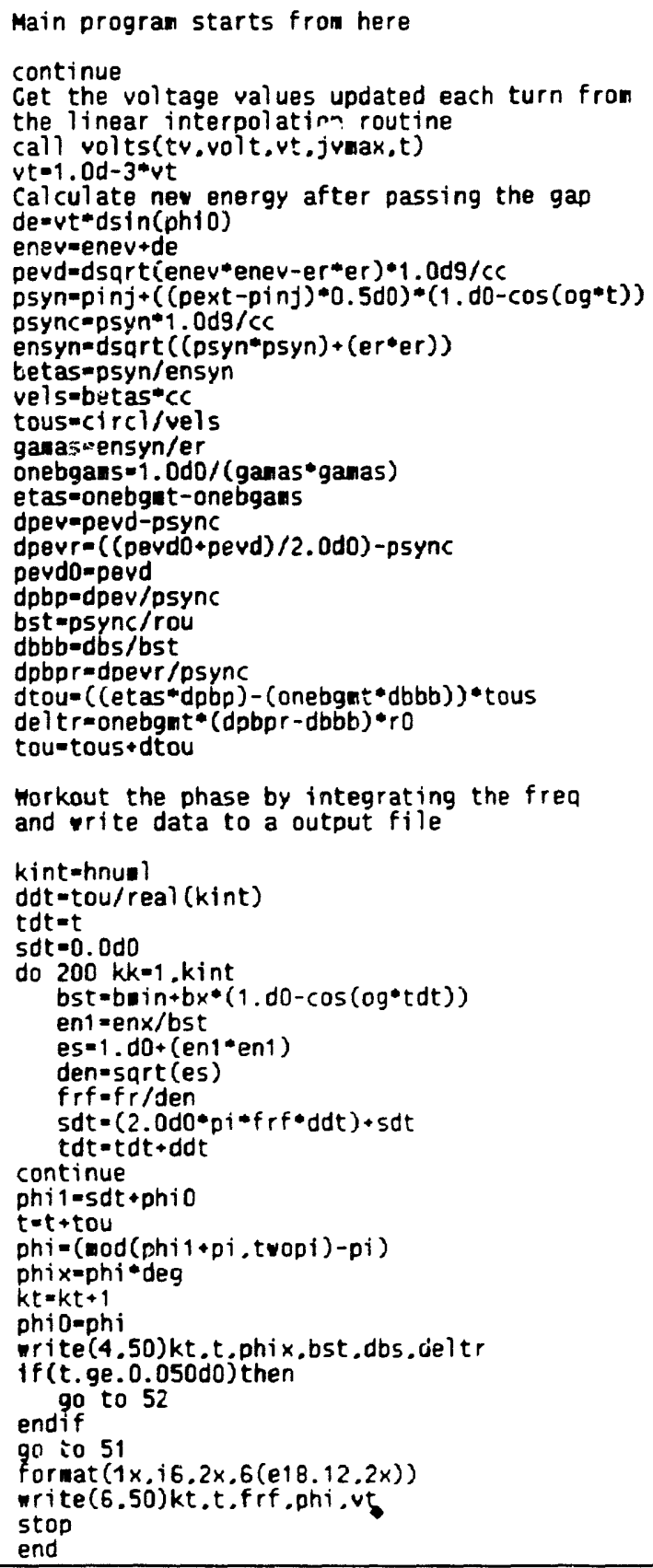 \\
\hline
\end{tabular}

Flgure 5. Fortran Code for Tracking One Particle. 


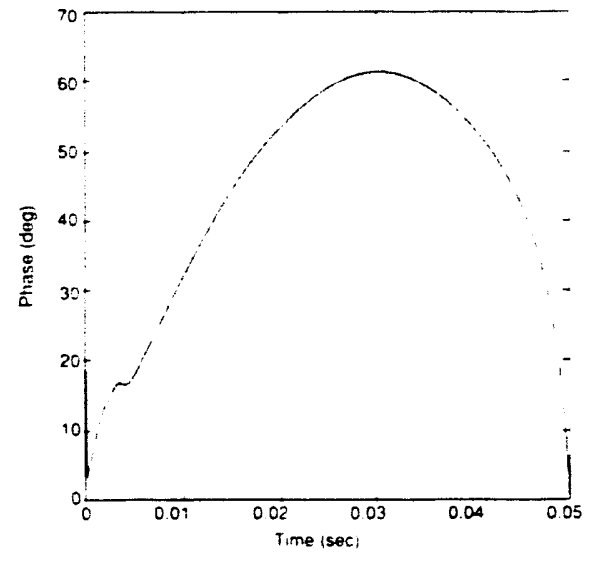

Figure 6. Synchronous Phase As Calculated from ESME.
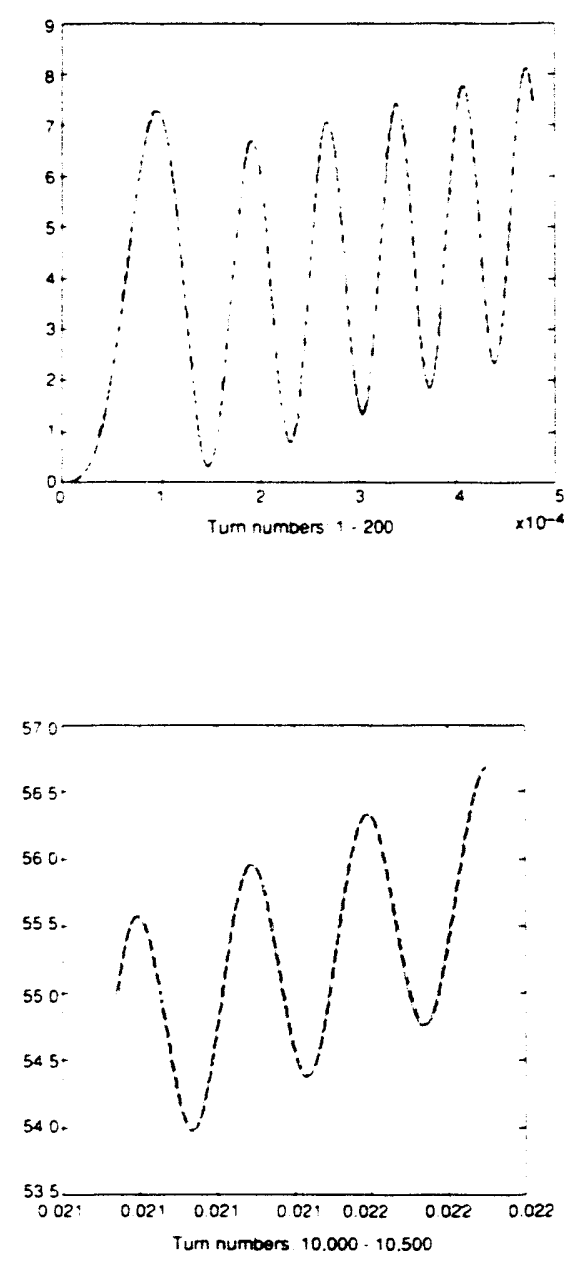

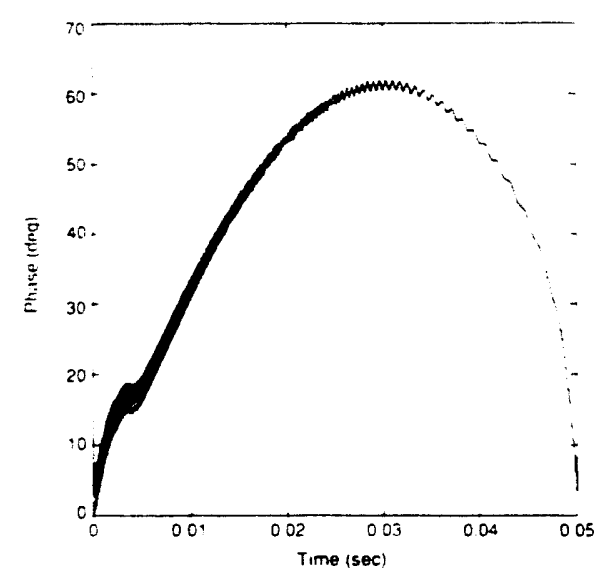

Figure 7. Particle Phase Predicted Using Thin Element Tracking Code.
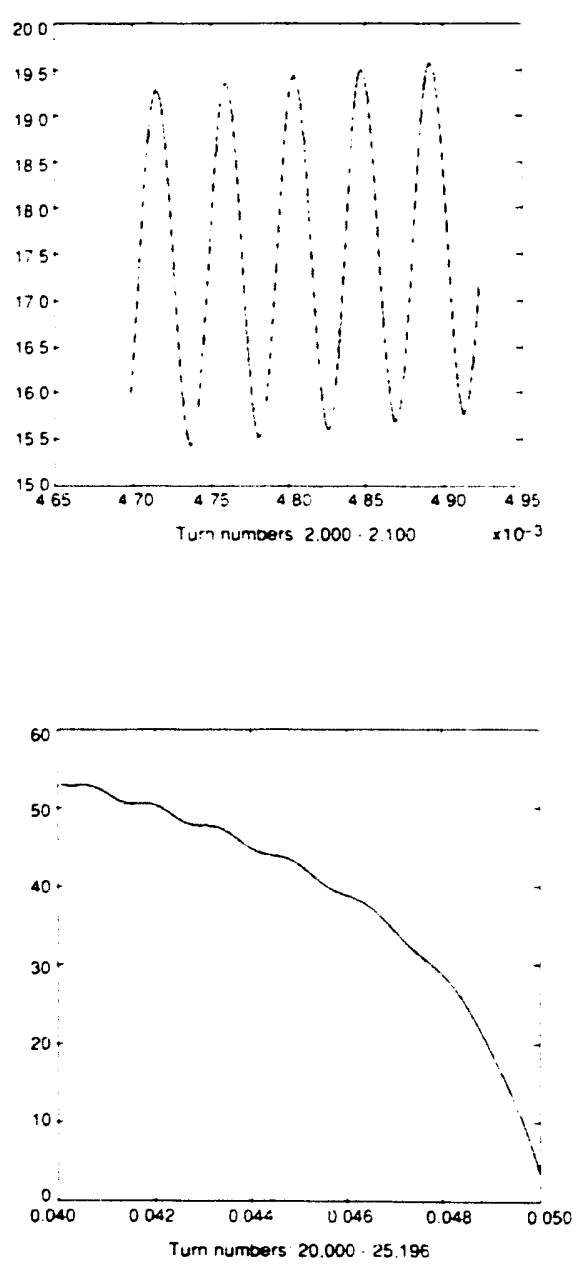

Figure 8. Phase Curves of Figures 4 and 7 Shown with Expanded Scale. 
Figures 9-12 compare the phase curves with field error. The field error was assumed to have Gaussian noise of bandwidth $1 \mathrm{KHz}$ with a normal distribution such that

$$
\delta B_{k}=2 \times 10^{-4} B_{k}(\text { rand }(\text { normal })) .
$$

The radial orbit errors are shown in Figures 13-16. Figure 17 shows the mean radial orbit with respect to the particle phase using Thin Element Tracking Code when the particle is tracked with an initial transverse displacement of $3 \sigma$ in both transverse planes. Clearly the envelope of the mean radial orbit curve matches well with Figures 15 and 16. The matching can also be seen in Figure 18, which is shown to compare Figure 17 with Figure 4. From this comparison we see that the betatron oscillations did not show large effects in the beam phase. In Figure 19 the tracking results are compared to Thin Element Tracking Codes for an initial phase, $\phi_{0}=10^{\circ}$.

Another useful test is seen in Figure 20. In this test tracking is done by injecting a particle at $\phi_{0}=0$ and by maintaining the bending field at $B_{\min }$ (minimum field at injection for a synchronous particle) and the if frequency at $f_{\min }$ (minimum frequency for a synchronous particle), throughout a $50 \mathrm{~ms}$ period. The plots of the phase curves must show no phase increase, as evident in Figure 20. Note that the scales are expanded to show a negligible difference with two curves. As we had expected, the particle gains no energy and hence returns to the accelerating gap with zero phase.

An if engineer would be interested to know about the beam behavior when one of the cavities breaks down. If it is not repaired soon enough there will be beam oscillations, as shown in Figure 21 . Figure 22 shows the plot with a voltage error of $1 \%, 50 \mathrm{KHz}$ bandwidth, and Gaussian noise with normal distribution.

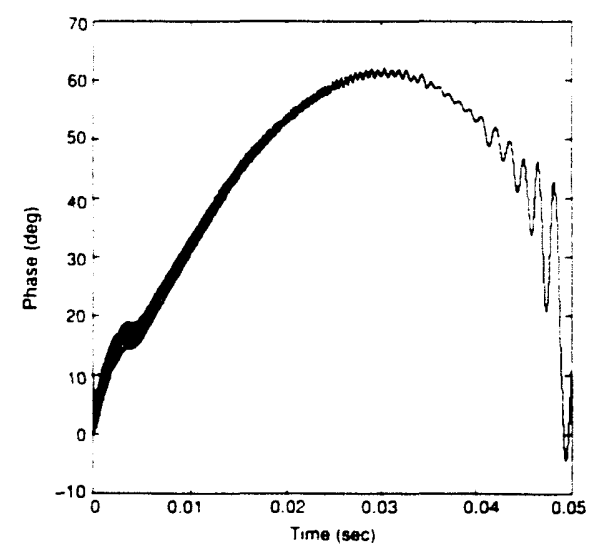

Figure 9. Time Variation of the B-fibld Error Signal.

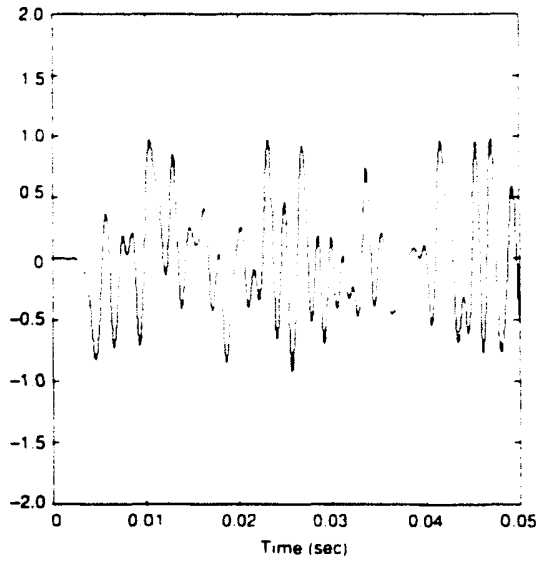

Figure 10. Particle Phase Predicted Using the Codes of Figure 5 with B-Error. 


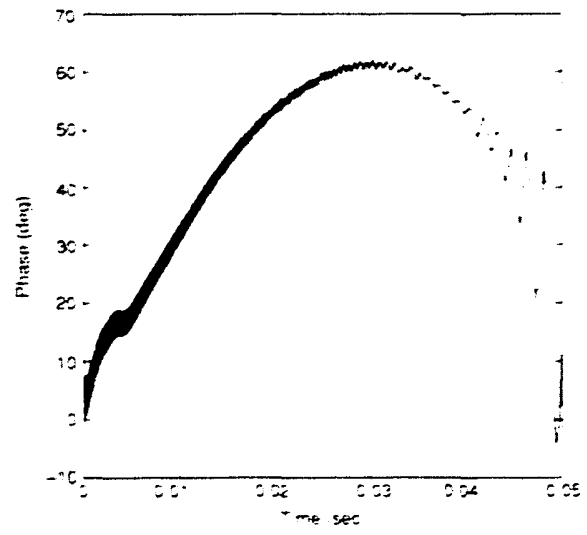

Figure 11. Particle Phase Predicted Using Thin Element Tracking Code with B-Error.
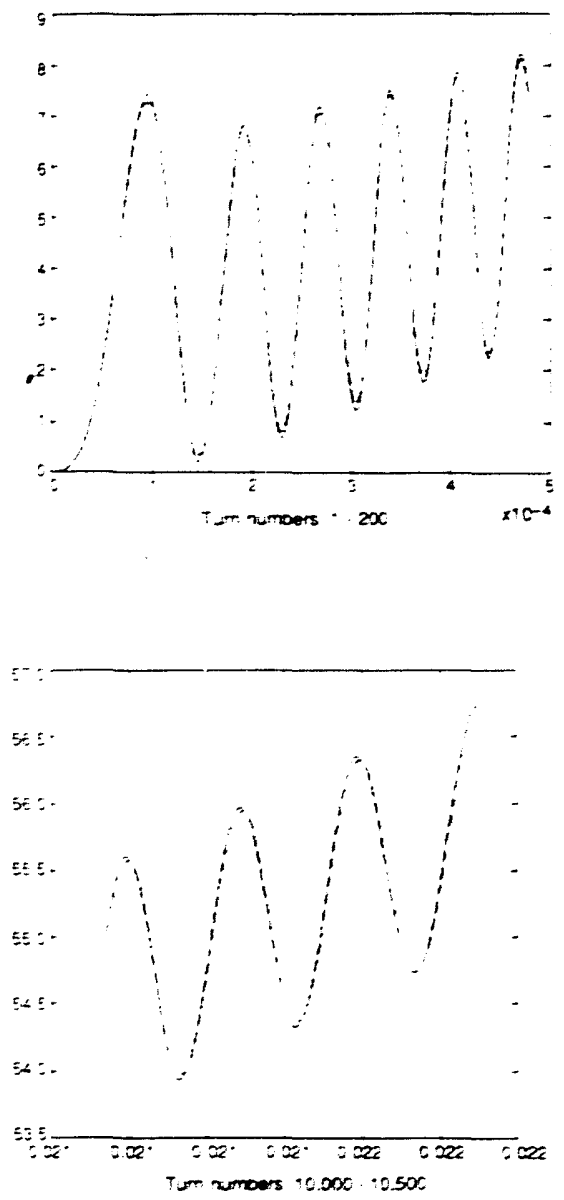
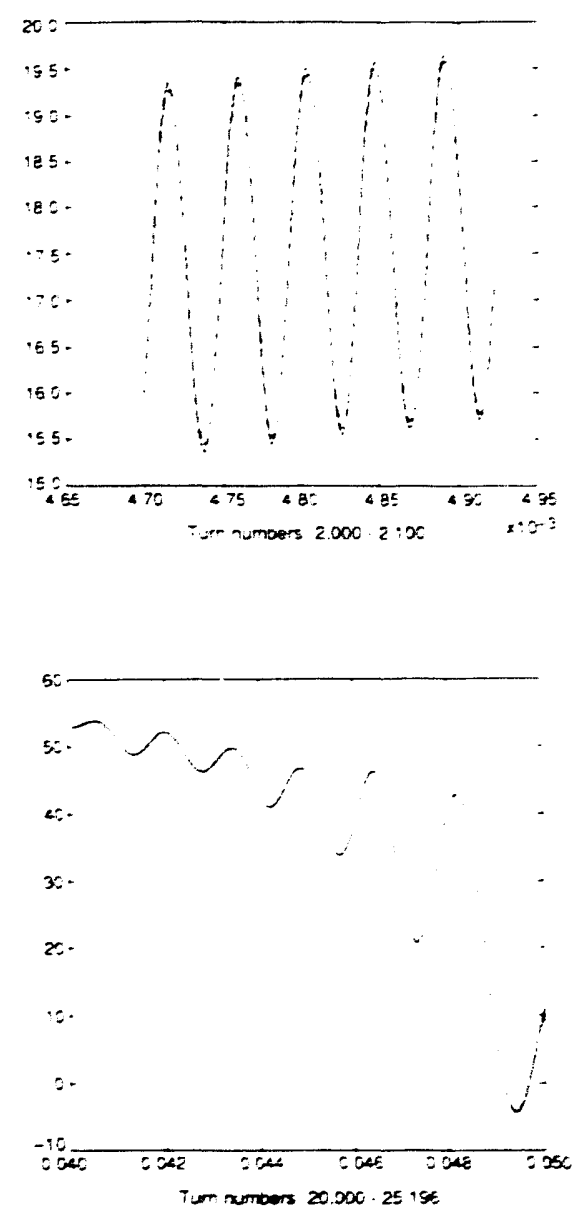

Figure 12. Phase Curves of Figures 10 and 11 Shown with Expanded Scale.

9 


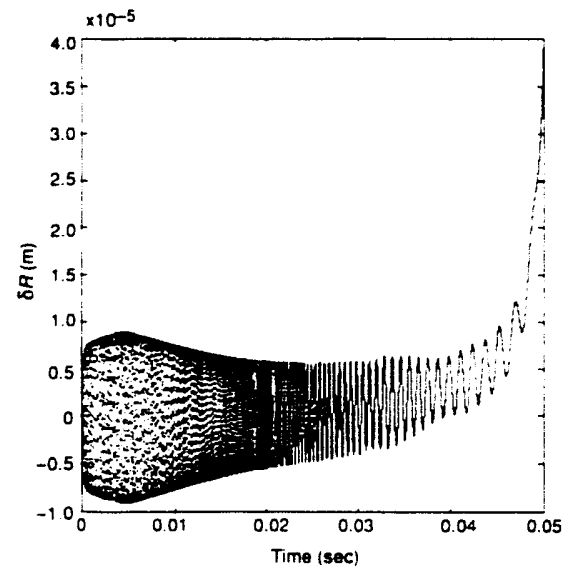

(a)

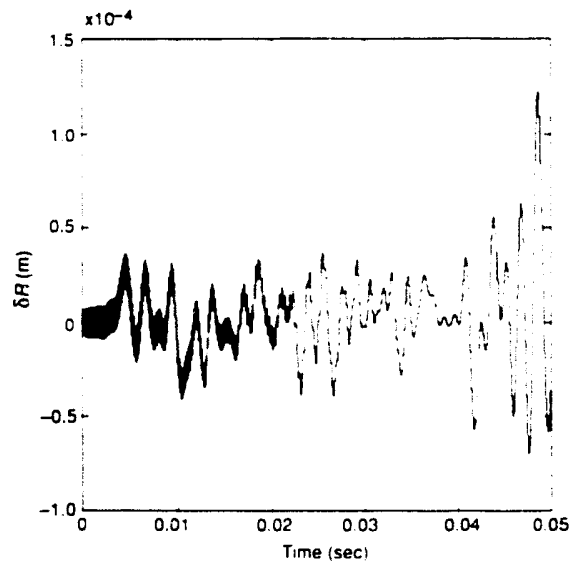

(b)

Figure 13. Man Radial Orbit :Shift Predicted Using Codes Shown In Flgure 5 with (a) $\delta B=0$ and (b) $\delta B$ as in Eq. (12).

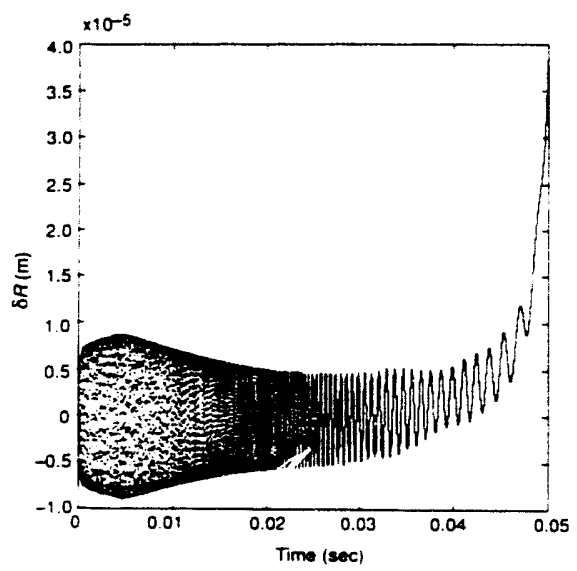

(a)

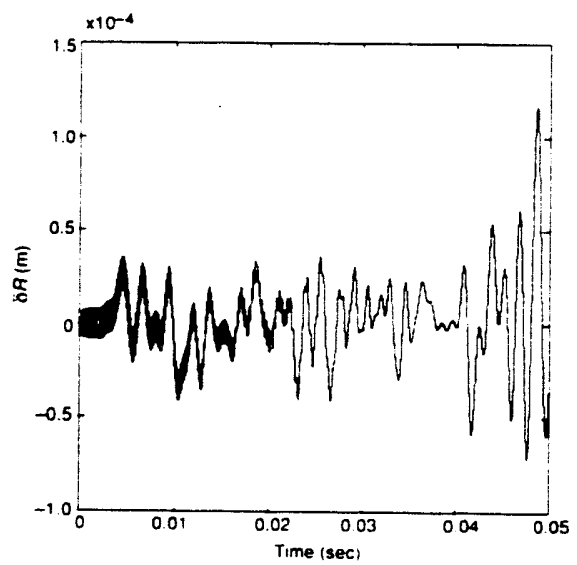

(b)

Figure 14. Moan Radlal Crblt Shift Predicted Using Thin Element Tracking Codo with (a) $8 B=0$ and (b) 83 as in Eq. (12).

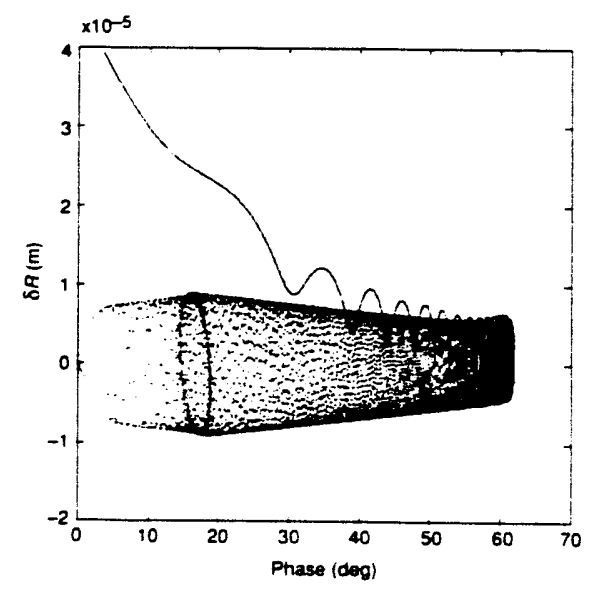

Flgure 15. Veriation of Mean Radial Orbit whth Respect to Particie Phase Uaing Codes Shown In Figure 5 (No Field Error Included).

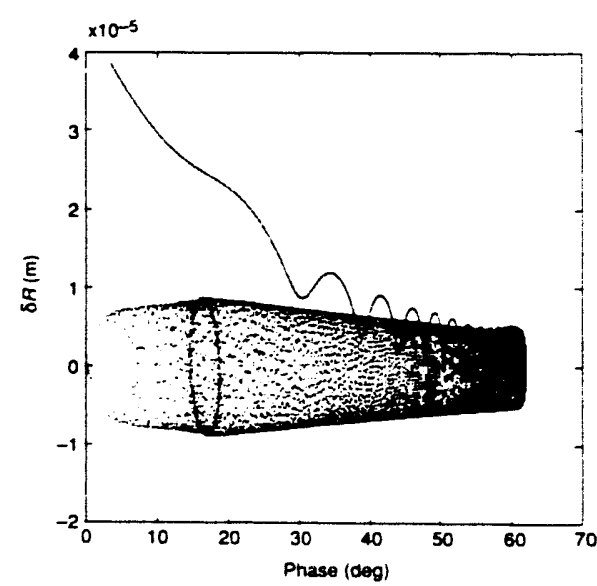

Figure 16. Variation of Mean Radial Orblt whth Respect to Particle Phase Using Thin Eloment Tracking Code (No Field Error included). 


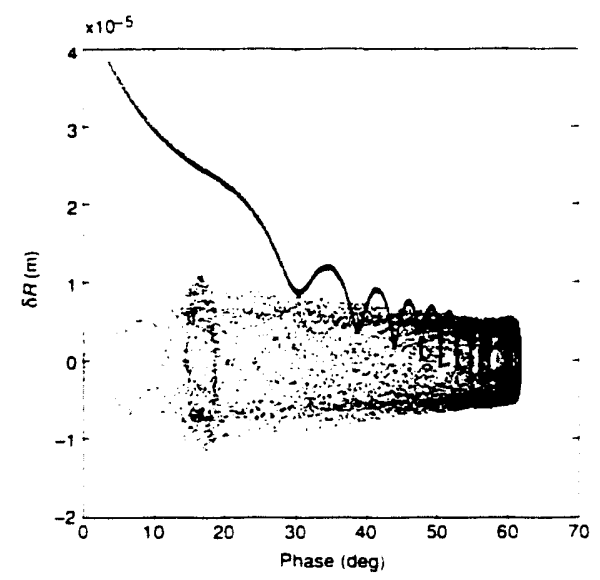

Figure 17. Variation of Mean Radial Orbit with Respect to Phase Using Thin Element Tracking Code When the Particle is Launched with 30 in Both Transverse Planes.
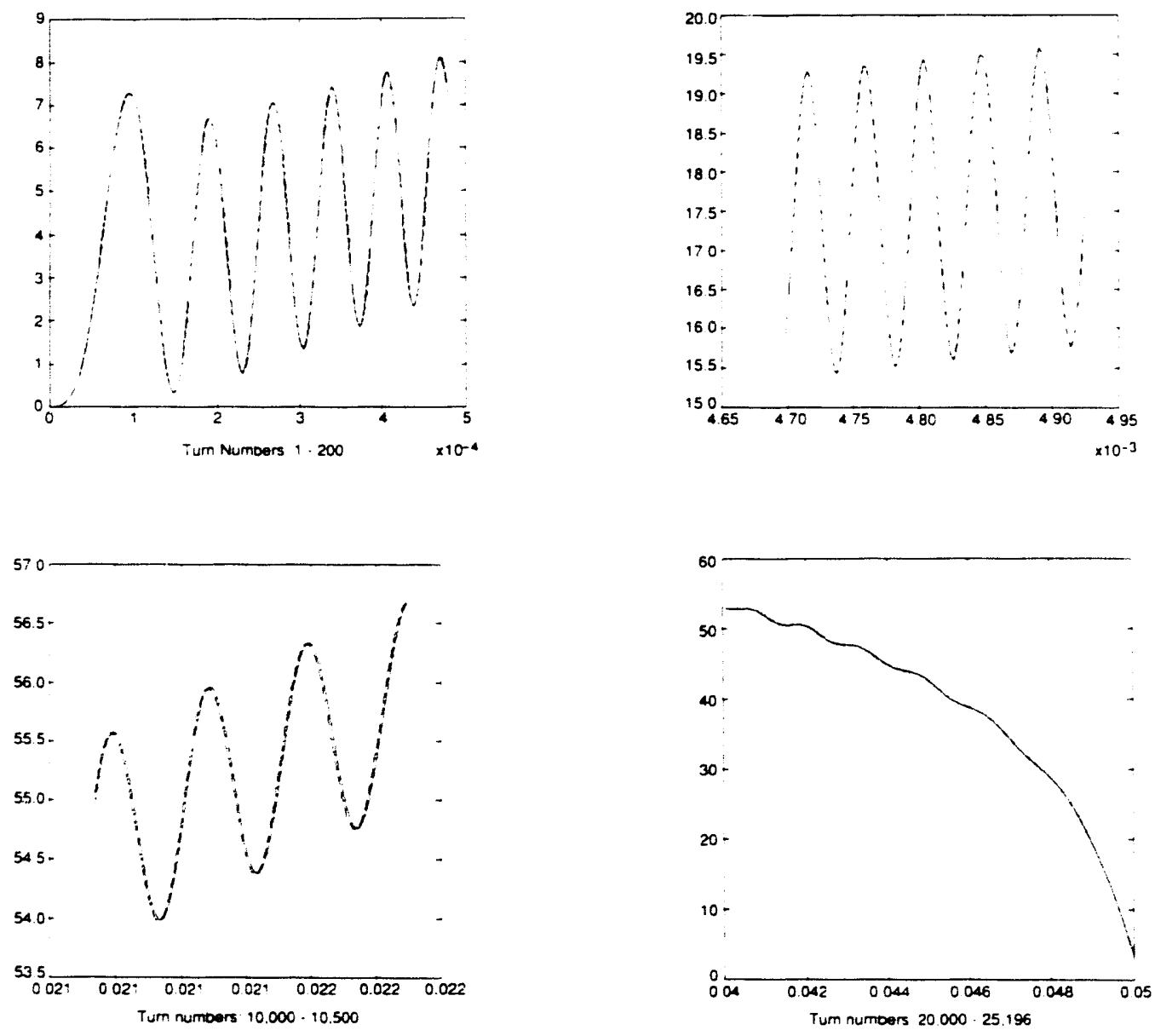

Figure 18. Phase Curves When the Particle Is Launched with $3 \sigma$ in Both Transverse Planes (Expanded Scale of Figures 4 and 17). 

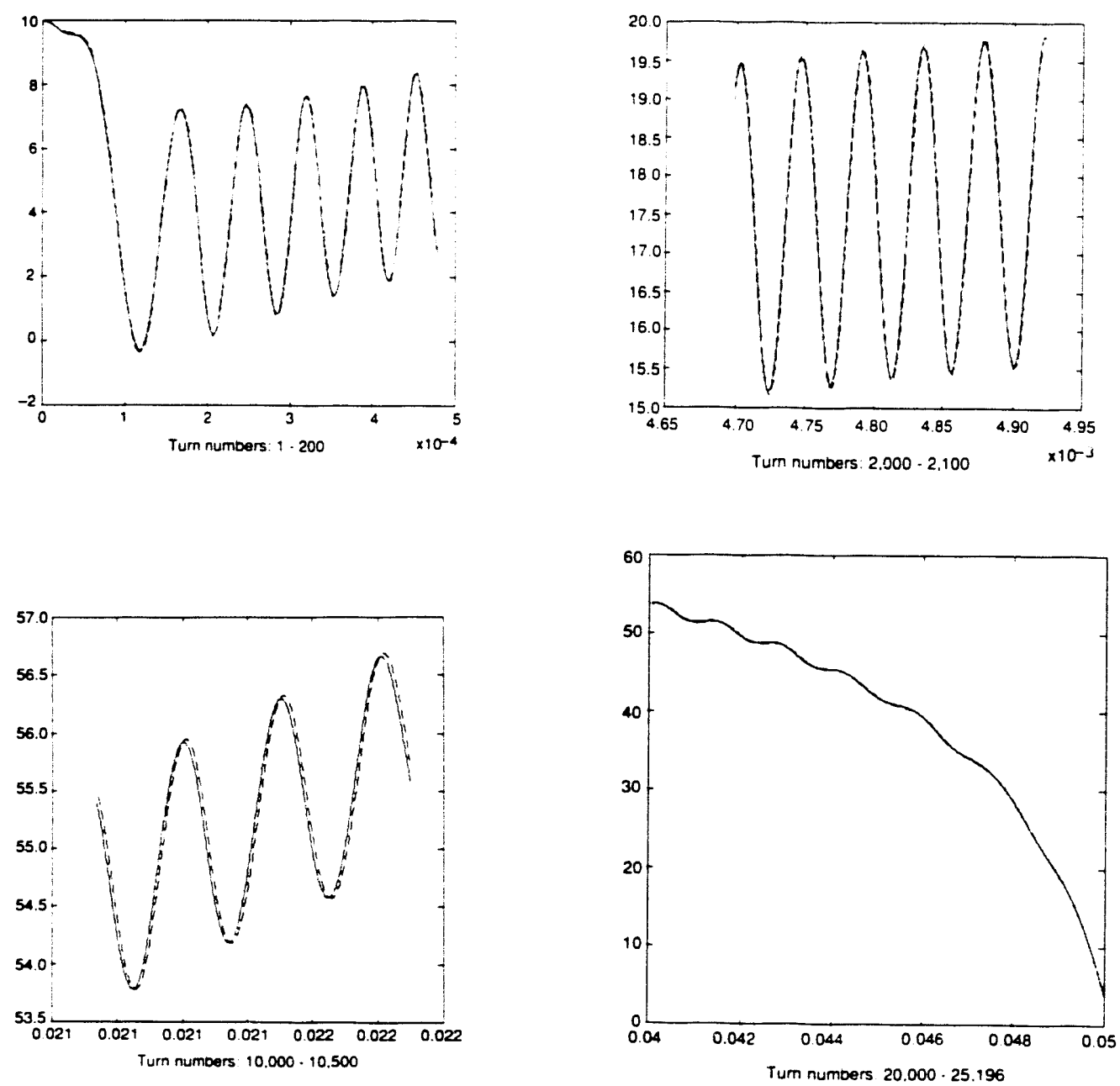

Figure 19. Phase Curves When the Particle is Launched at +10 deg. 

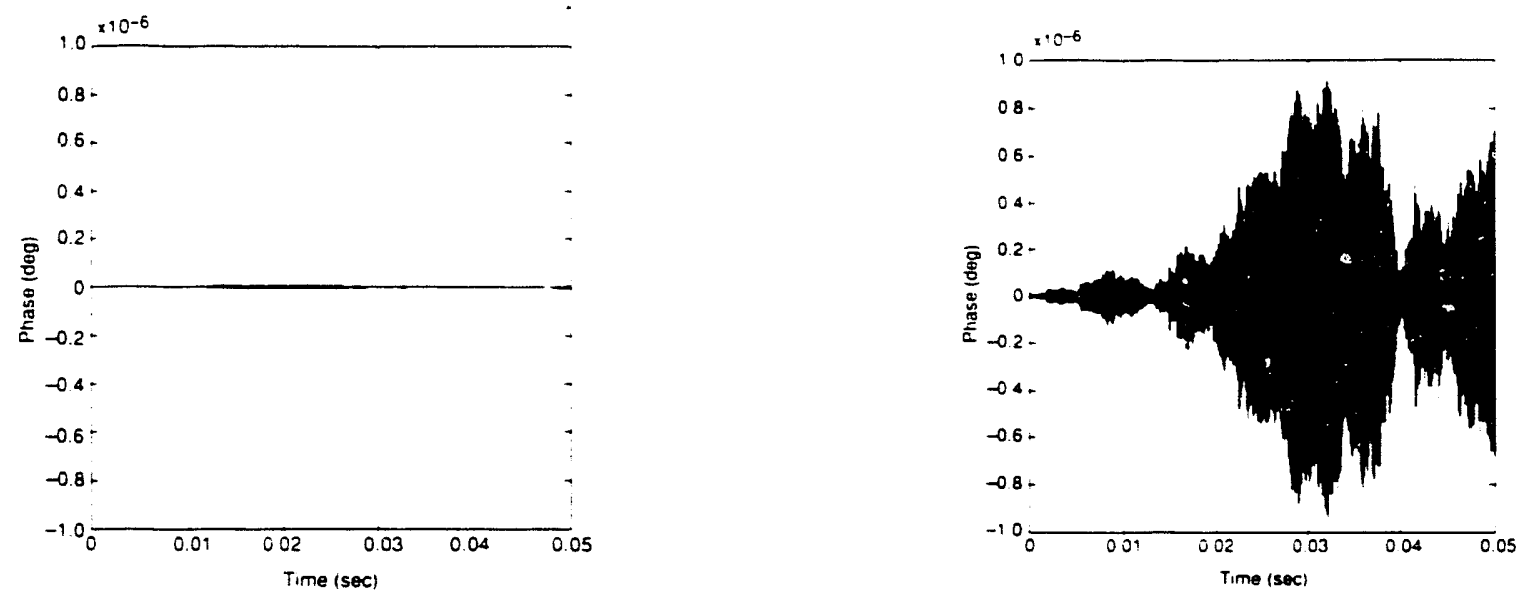

Figure 20. Particle Phase with Respect to Time When the B-Fleld ls Held at Minimum $B$ for 50 ms (a) Using the One-Particle Tracking Code and (b) Using the Thin Element Tracking Code.

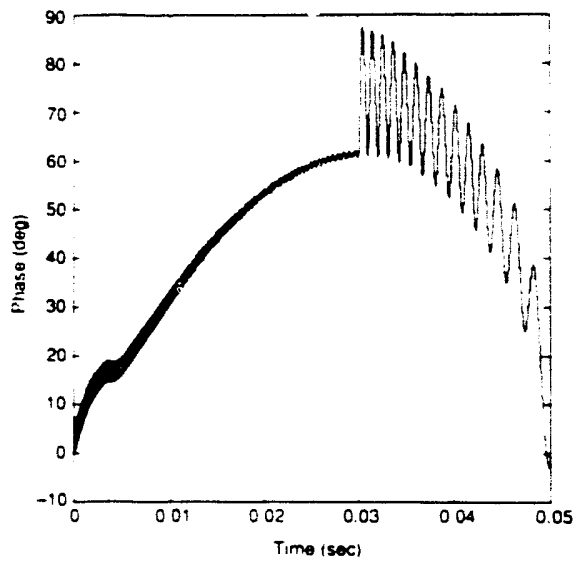

Figure 21. Phase Curve When 1 out of 12 Cavities Breaks Down at $30 \mathrm{~ms}$.

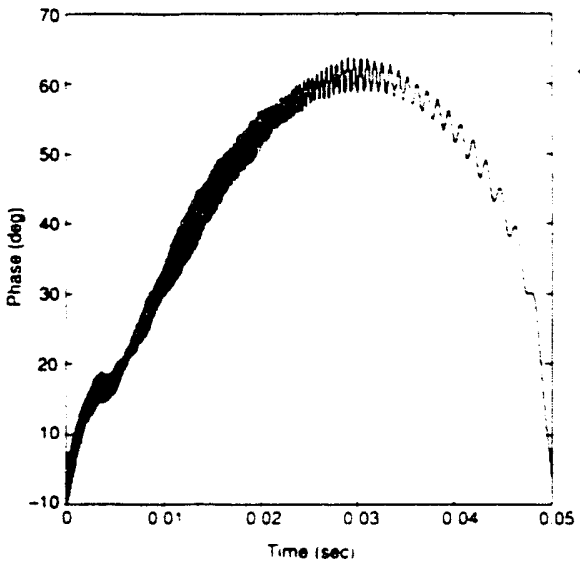

Figure 22. Phase Curve with a Voltage Error of $50 \mathrm{KHz}$ Bandwidth and $1 \%$ Amplitude Deviation.

\subsection{RF BEAM FEEDBACK LOOPS}

As discussed earlier, the code was written mainly to study the effect of $\mathrm{rf}$ feedback loops on the beam. Conceptually, as seen from the beam model in Table 1, rf system feedback loops are very simple. An if power amplifier drives the cavity, and subsequently a high voltage appears on the gap. The amplitude of this voltage is programmed and is controlled by a feedback loop to supply the required voltage to the gap. as schematically shown in Figure 23. To derive $v_{k}$, a combined power amplifier and the cavity model must be included in the model. The frequency and phase of the if signal are generally controlled with feedback loops from the beam to the frequency generator and a phase shifter. Feedback controllers can be included to stabilize the loops. With a high-power if system model in the loop we can obtain the desired frequency and phase, $f_{k}$ and $\phi_{k}$, at the gap. Thus beam oscillations can be studied with the feedback loops and for different types of controllers. If the beam frequency must be synchronized with an external reference, then a synchronization loop can be inserted to modulate the frequency and phase of the if generator. If phase-locking with the higher energy accelerator is required, then the synchronization loop can be turned on at any given time in the acceleration cycle. 


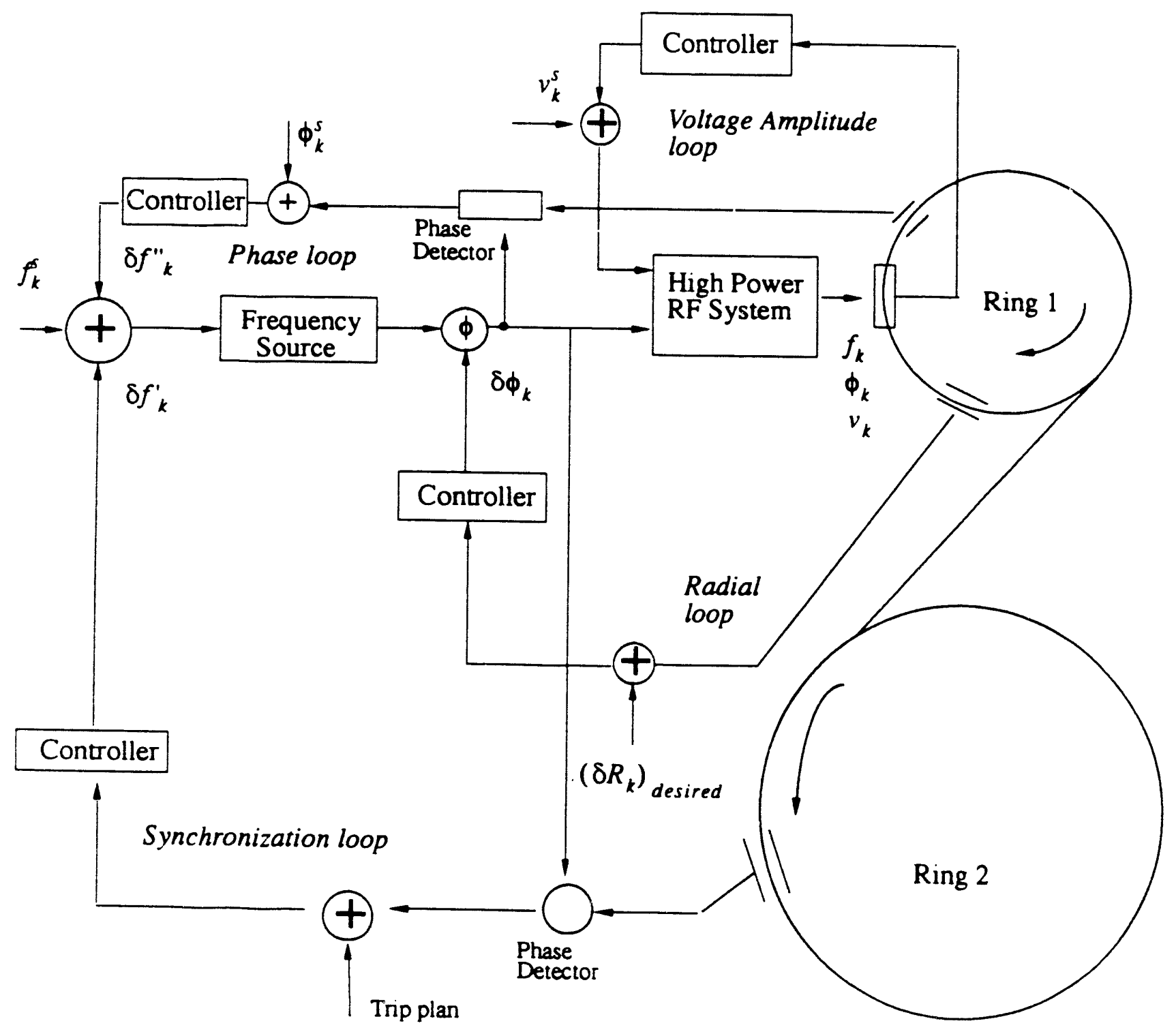

Figure 23. Conceptual Dlagram of the Various if Beam Feedback Loops with One if Cavity. 
The beam phase loop shown in Figure 23 will hielp to set the synchronous phase to a desired value provided we can allow the beam to breathe radially. Since the beam tube is always confined to a small size, a radial loop is required in the system. At this stage we cannot tell to what extent we can control the synchronous phase without modeling with the beam in the orbit. In the same way, if the synchronization loop is closed throughout the acceleration of the low-energy ring, then the interaction is even more difficult to understand. Furthermore, it would be possible to connect the radial loop to modulate the amplitude of the gap voltage, since the change in the amplitude of the gap voltage changes the energy of the beam-in effect, changing the radial position. All the controllers mentioned in Figure 23 can be contained in a single equation, if appropriately designed to take beam physics into account. This global controller should be able to take into account the noise in the system at different points in the feedback loop and to accelerate the beam so that it will reach the destination ring at the precise time and energy. Our work is progressing in this direction. 


\section{ACKNOWLEGEMENTS}

The author is grateful to Bob Webber for inspiring me to follow this route in obtaining a simple tracking model. Many useful discussions and simulation results provided by Shinji Machida using Thin Element Tracking Code are gratefully acknowledged. Excellent computer support provided by P. Ravishankar is also appreciated. 


\section{REFERENCES}

1. C. Bovet el al., "A Selection of Formulae and Data Useful for the Design of A.G. Synchrotrons," CERN Report No. CERN/MPS-SI/Int. DL/70/4, April 23, 1970.

2. S. Machida, "Modification of TEAPOT to Include Acceleration," ADOD notes, ADOD-028Q, SSC Laboratory, January 1990. 

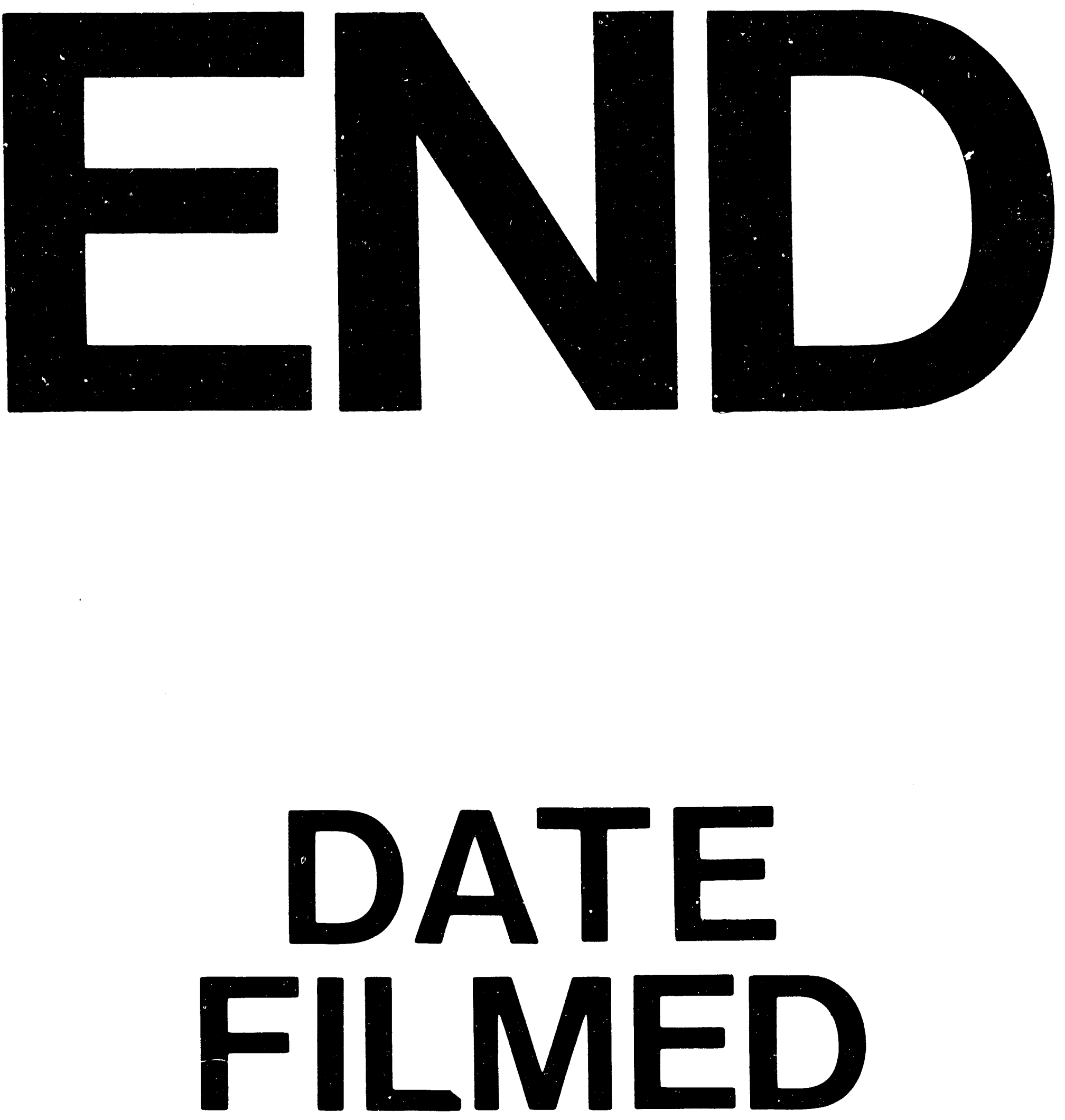

†

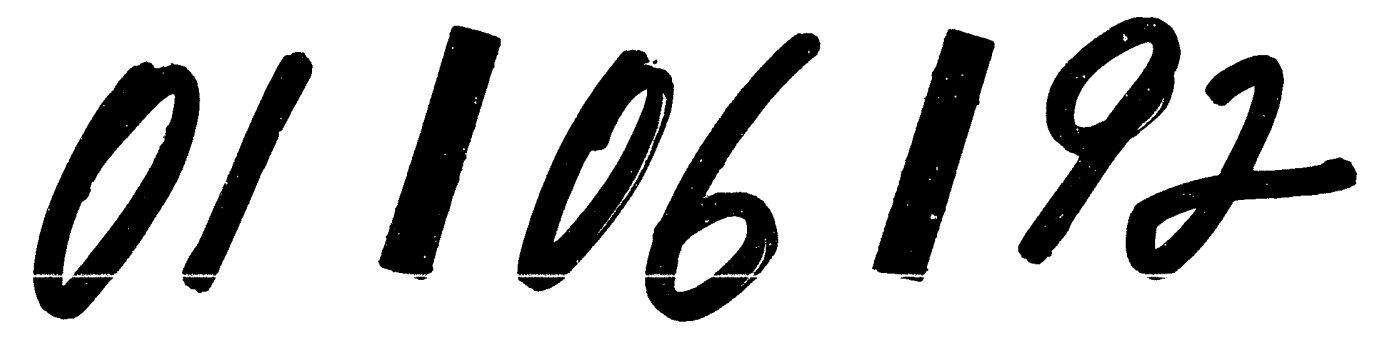


